\title{
Heparin Microlslands to Promote Enhanced Diabetic Wound Healing Outcomes
}

\author{
Lauren Pruett ${ }^{1}$, Christian Jenkins ${ }^{1}$, Neharika Singh ${ }^{1}$, Katarina Catallo ${ }^{1}$, Donald Griffin ${ }^{1,2}$ \\ ${ }^{1}$ Department of Biomedical Engineering, University of Virginia, Charlottesville, VA \\ ${ }^{2}$ Department of Chemical Engineering, University of Virginia, Charlottesville, VA
}

\begin{abstract}
A powerful tool to improve tissue integration with biomaterial scaffolds for the regeneration of damaged tissues is to promote cell migration using chemotactic gradients of growth factors. This approach has been realized by the exogenous delivery of growth factors, which unfortunately also limits the scaffold's ability to meet each wound's unique spatial and temporal regenerative needs. To address this limitation, we present a new approach to gradient generation by incorporating heparin microislands, which are spatially isolated heparincontaining microparticles that create chemotactic microgradients through reorganization of endogenous local growth factors. We incorporated heparin microislands within microporous annealed particle (MAP) scaffolds, which allows us to tune their incorporation ratiometrically to create a heterogenous microenvironment. In this manuscript, we demonstrate the ability of heparin microislands to organize uniform growth factors into spontaneous microgradients and control downstream cell migration in vitro. Further, we present their ability to significantly improve wound healing outcomes (epidermal regeneration and vascularization) in a diabetic wound model relative to two clinically relevant controls.
\end{abstract}

Keywords: Porous hydrogel, diabetic wound healing, microgradient, heparin microislands 


\section{Introduction}

Growth factors are key regulators of each stage of wound repair, including cellular migration, proliferation, angiogenesis, and extracellular matrix remodeling ${ }^{1}$. Their potential for accelerating wound healing has led to a long history of research efforts focused on biomaterial scaffold-mediated delivery of a range of growth factors (e.g. PDGF, VEGF, EGF, FGF) ${ }^{2-4}$. Importantly, despite FDA approval, growth factor delivery has been limited in clinical translation by safety concerns and cost-effectiveness ${ }^{2,3,5,6}$.

In uninjured tissues, growth factors are an essential component of the instructional microenvironment and their spatial organization within that microenvironment is shaped, in part, by their affinity interactions with the structural components of the extracellular matrix $(E C M)^{1,2}$. Specifically, the ECM can regulate growth factor movement and create chemotactic gradients for spatio-temporal regulation of cell migration ${ }^{2}$. Heparin, the most highly charged glycosaminoglycan in human ECM, is an important regulator of growth factor localization and retention due to its high binding affinity with many growth factors via electrostatic interactions, including growth factors that are important for wound repair (e.g. Vascular Endothelial Growth Factor [VEGF]) ${ }^{7-9}$. Due to its high affinity for many growth factors, heparin has been incorporated homogeneously within biomaterial scaffolds to provide chemotactic bioactivity via controlled exogenous growth factor release ${ }^{7,8}$.

In addition to heparin-based controlled release of growth factors, multiple strategies have been developed to harness chemotactic gradients within biomaterial scaffolds, including those incorporating pre-programmed degradable carriers (e.g. GF-loaded PLGA nanoparticles) or advanced fabrication techniques (e.g.

photopatterning $)^{10}$. However, these techniques are limited by the macroscale (i.e. non-physiologic ${ }^{2}$ ) nature of their gradients ${ }^{11}$ and an inability to dynamically alter their signaling in response to tissue formation/remodeling. By contrast, in this manuscript we present an exciting heterogeneous heparin incorporation technique using spatially isolated hydrogel microspheres with covalently immobilized heparin, or heparin "micro-islands" ( $\mu$ lslands), that offer growth factor-mediated bioactivity, including chemotaxis, that is not reliant on exogenous growth factors. Our heparin $\mu$ lslands create chemotactic gradients through reorganization of endogenous local growth factors.

To incorporate heparin $\mu$ lslands in a format that allows cells to freely respond to chemotactic gradients, we took advantage of an injectable biomaterial platform, microporous annealed particle (MAP) scaffolds ${ }^{12}$. MAP scaffolds are composed of micron-scale spherical building blocks (microspheres) that can be mixed ratiometrically with heparin $\mu$ lslands to achieve controlled heterogeneity. Further, MAP is assembled via covalent inter-microsphere bonding (i.e. annealing) in situ to form structurally stable scaffolds with cell-scale microporosity. In addition to observing the spontaneous reorganization of uniformly applied growth factors into micro-scale (i.e. physiologically relevant) gradients around heparin $\mu$ lslands, we also validated their impact on cell chemotaxis and whole tissue regenerative behavior.

Specifically, we chose to focus the application of this phenomenon on diabetic wounds, which pathologically suffer from both a lack of organized tissue regeneration and growth factor retention ${ }^{5,13,14}$. Diabetic wounds result in approximately 130,000 lower limb amputations each year and affect $15 \%$ of diabetic patients in the U.S. ( 10\% of U.S. population has diabetes $)^{15,16}$. Clinically, these wounds present a challenging healing environment that suffers, in part, from poor growth factor regulation ${ }^{5,6,13,14}$. Despite FDA approval of several therapies including decellularized matrices and recombinant growth factor (e.g. PDGF) delivery, their clinical use remains limited and even after treatment half of these wounds never heal ${ }^{6,13,14,17}$. We hypothesize that heparin $\mu$ Islands, with their unique ability to concentrate growth factors into spontaneous microgradients, can improve the healing of these wounds. Therefore, we chose to test heparin $\mu$ lslands in a relevant diabetic wound healing animal model ${ }^{18}$ using the most common class of advanced wound treatment (decellularized tissue $^{6,19}$ ) as our clinically-relevant control. 

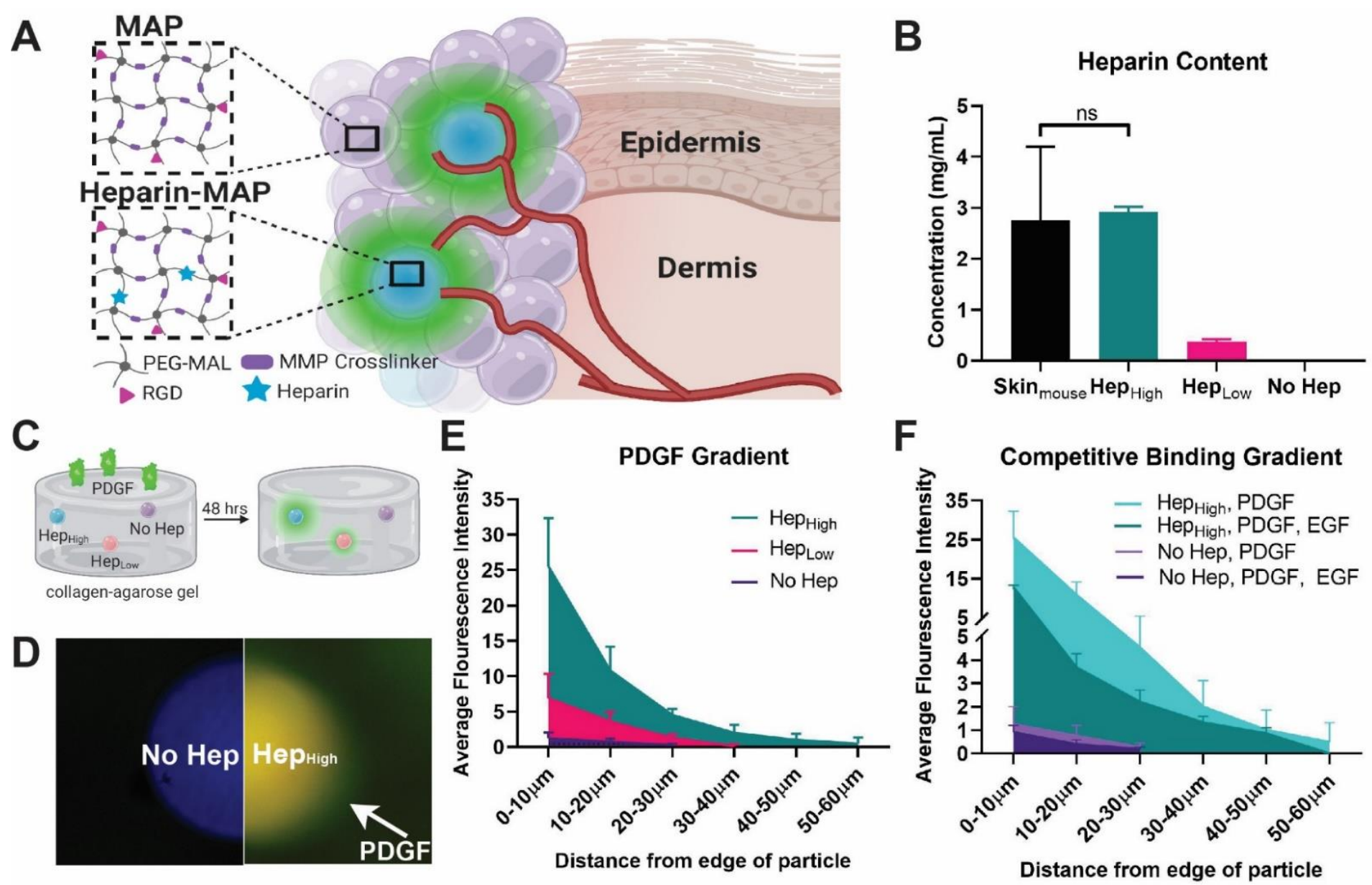

Figure 1. Synthesis and Gradient Characterization of Heparin $\mu$ lslands. A) Particles were composed of a PEG-maleimide backbone and MMP-2 cleavable crosslinker with an RGD cell adhesive peptide with or without thiolated heparin. Small amounts of heparin particles ( $\mu$ lslands) were mixed with non-heparin particles to generate growth factor gradients. B) Heparin concentration within the particles was matched to mouse skin (HepHigh) and one tenth of mouse skin (HepLow). C) To test for gradient formation around our heparin $\mu$ islands, we used a well-based assay with individual particles embedded within a collagen-agarose scaffold before introduction of a solution of biotin-labeled PDGF. D) After fixation, fluorescent Streptavidin revealed a PDGF gradient (green) around the heparin $\mu \mathrm{lsland}(\mathrm{red})$ and was absent around particle without heparin (blue). E) Quantification of fluorescence of concentric rings surrounding the microgels confirmed the presence of a gradient of PDGF. F) Gradient formation was maintained at a lower magnitude when microgels were incubated with PDGF and a competitive binding protein (EGF).

\section{Particle building blocks to create a heterogeneous porous scaffold}

Using our MAP scaffolds ${ }^{12}$ as a platform technology, we took advantage of the ability to mix and match particle populations to create ratiometrically-controlled heterogenous scaffolds while maintaining an injectable format. To design instructional interaction with chemokines, we chose to focus on heterogeneously distributed heparin particle populations (heparin $\mu$ lslands). We hypothesized that heparin $\mu$ lslands would locally sequester growth factors released endogenously in a wound environment and generate functional microscale gradients. Three particle populations with variable heparin concentrations (Hep High, HepLow, and no Hep) were produced using a previously published high-throughput microfluidic method ${ }^{20}$ to isolate heparin concentration as the only changing variable between particle types by providing uniform geometric (diameter: $90 \mu \mathrm{m}$, Supplemental Fig. 6) and mechanical (Young's modulus: 18kPa, Supplemental Fig. 5) properties. The particles were composed of a synthetic hydrogel network of 4-arm poly(ethylene glycol) maleimide backbone crosslinked with a peptide sequence optimized for enzymatic resorption by matrix-metalloprotease-2 (MMP-2) and covalently bonded to an RGD cell adhesive peptide ligand (Fig. 1A). A custom heterofunctional 4-arm PEG

maleimide/methacrylamide macromer was incorporated to facilitate scaffold annealing ${ }^{21}$. With consideration for our future diabetic wound healing assays, thiolated heparin ${ }^{22}$ was incorporated into the heparin $\mu$ lslands at a concentration chosen to mimic mouse skin (Hep High $_{\text {) }}$ and one-tenth mouse skin (HepLow) (Fig. 1B). 


\section{Spontaneous microgradients from uniform growth factor}

To validate our hypothesis that heparin $\mu$ lslands can sequester and organize uniform growth factor distributions into microscale gradients, we embedded them within a collagen-agarose gel and incubated them with a solution of platelet-derived growth factor (PDGF) (Fig.1C). We chose PDGF for both its importance in wound healing and its known interactions with heparin ${ }^{3,9}$. After 48 hours of incubation with biotinylated PDGF, the gels were fixed and stained with fluorescent streptavidin. A gradient was visibly present around the heparin $\mu$ lslands and was absent around particles without heparin (Fig. 1D). Quantification of relative gradient strength showed that increased levels of PDGF were detected out to $60 \mu \mathrm{m}$ around Hep $\mathrm{High}_{\mu} \mu$ slands and $40 \mu \mathrm{m}$ around Hep Low $\mu$ lslands (Fig. 1E). To test the impact of competitive binding on spontaneous gradient formation (a complication expected in vivo), the same PDGF assay was conducted again for the HepHigh particles with the additional complexity of added non-biotinylated Epidermal Growth Factor (EGF), which is also known for heparin-specific interactions. Spontaneous gradient formation was still observed around the HepHigh $\mu$ lslands out to $60 \mu \mathrm{m}$, but at lower magnitude than PDGF alone (Fig. 1F). Thus, with these in vitro assays, we were able to confirm an affinity-based structure-function relationship for heparin $\mu$ lslands generating microscale gradients. These results prompted further investigation of potential effects on in vitro cell migration.

A

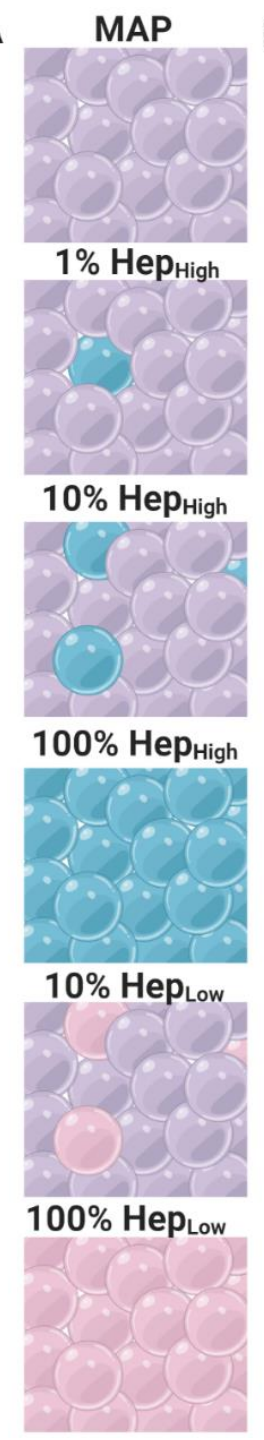

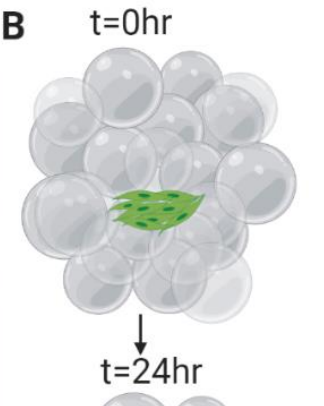

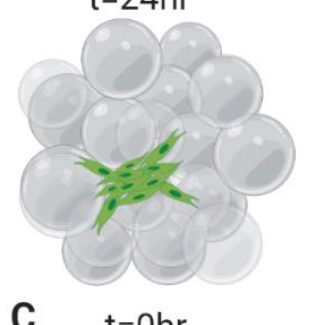

C
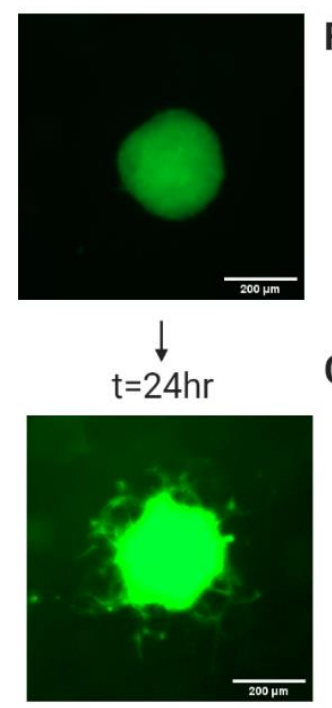

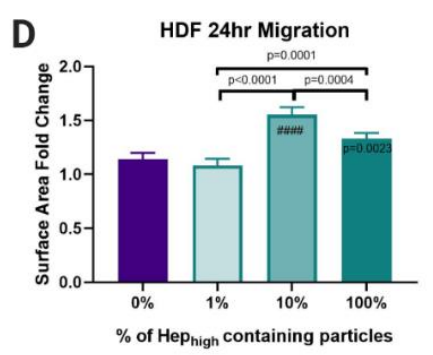

E

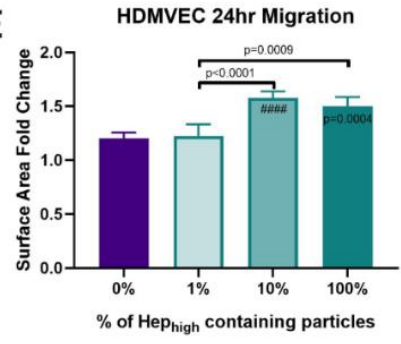

$\mathbf{F}$

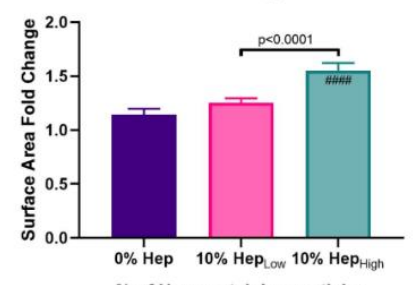

G

$\%$ of Hep containing particles

HDMVEC 24hr Migration

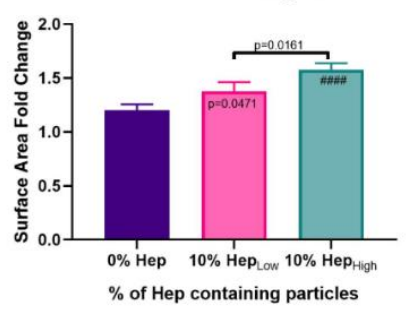

Figure 2. Cellular Migration. A) Six gel conditions were compared with varying concentrations and percentages of heparin particles. B) A spheroid migration assay was used to quantify cell migration over 24 hours. C) Representative images of spheroids at 0 and 24 hrs. D) Quantification of cell migration showed the 10\% HepHigh $\mu$ lslands exhibited the highest migration of Human Dermal Fibroblasts (HDFs) and E) Human Dermal Microvascular Endothelial Cells (HDMVECs) compared to all other groups with the same concentration of heparin, but different spacing. F) The 10\% HepHigh $\mu$ Islands significantly outperformed the $10 \%$ HepLow $\mu$ lslands for HDFs and G) HDMVECs confirming the concentration of heparin and the spacing of heparin particles effect cell migration. Statistics: ANOVA, Multiple comparisons post-hoc tests (Tukey HSD). $\mathrm{N}=4$. Significance inside of bars represents comparison to no hep gels. \#\#\# is $p<0.0001$. 


\section{Cell migration is dependent on particle spacing and heparin concentration}

To further investigate the potential functional impact of heparin $\mu$ lslands, we employed an in vitro spheroid migration assay developed for hydrogel constructs ${ }^{23}$ (Fig. 2B-C). Due to the potential for subsequent use in a diabetic dermal wound healing model, we chose two primary cell types relevant in dermal wound healing: human dermal fibroblasts and human dermal microvascular endothelial cells. Both particle ratio (heparin $\mu$ slands to no hep particles) and heparin concentration were varied to determine the effects of the heterogenous distribution of heparin $\mu$ lslands (Fig. 2A). Notably, both ratio and concentration affected migration behavior for both cell types. Specifically, for ratios of heparin particles, we observed an interesting impact of heterogeneity, where just $1 \% \mathrm{Hep}_{\text {High }}$ $\mu$ lslands had no noticeable difference in migration compared to no heparin gels, but $10 \%$ had the greatest migration compared to all other groups (Fig. 2D-E). However, using just $10 \%$ Hep $_{\text {Low }}$ particles only resulted in a slight increase in migration compared to no heparin (Fig. 2F-G), confirming that heparin concentration remains a critical factor. The cell migration in the $10 \% \mathrm{Hep}_{\text {High }} \mu$ Islands $_{\text {s }}$ was significantly greater than $100 \%$ Hep (Supplemental Figs. 9,10), verifying that heterogenous microenvironments promote the spatial cues necessary to increase migration without needing external factors (e.g. growth factors). The enhanced migration seen with $10 \% \mathrm{Hep}_{\text {High }} \mu$ Islands in HDFs and HDMVECs prompted the use of this group for the diabetic wound healing studies.

A

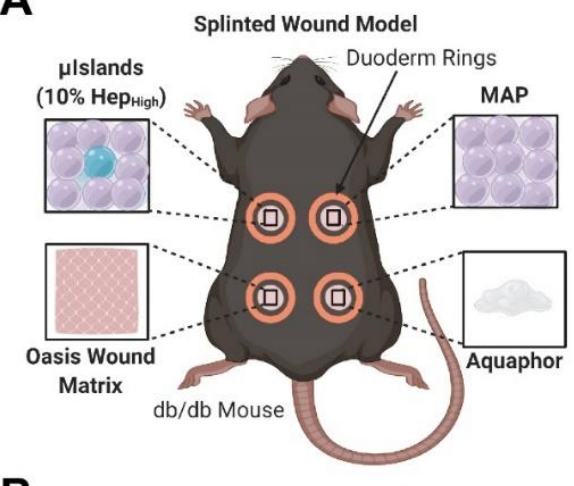

B

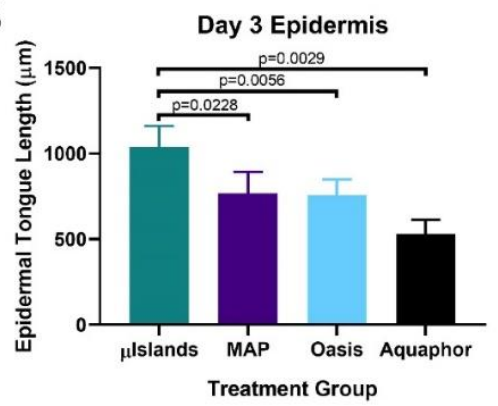

C

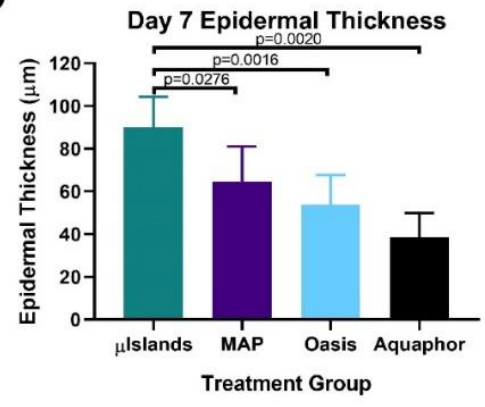

D
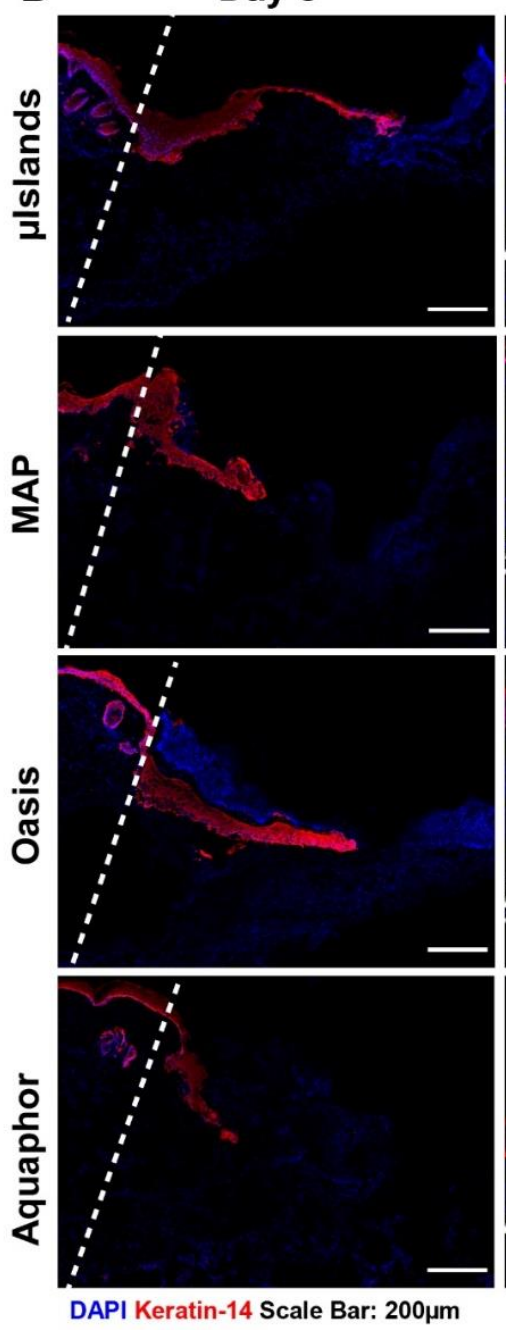

Day 7
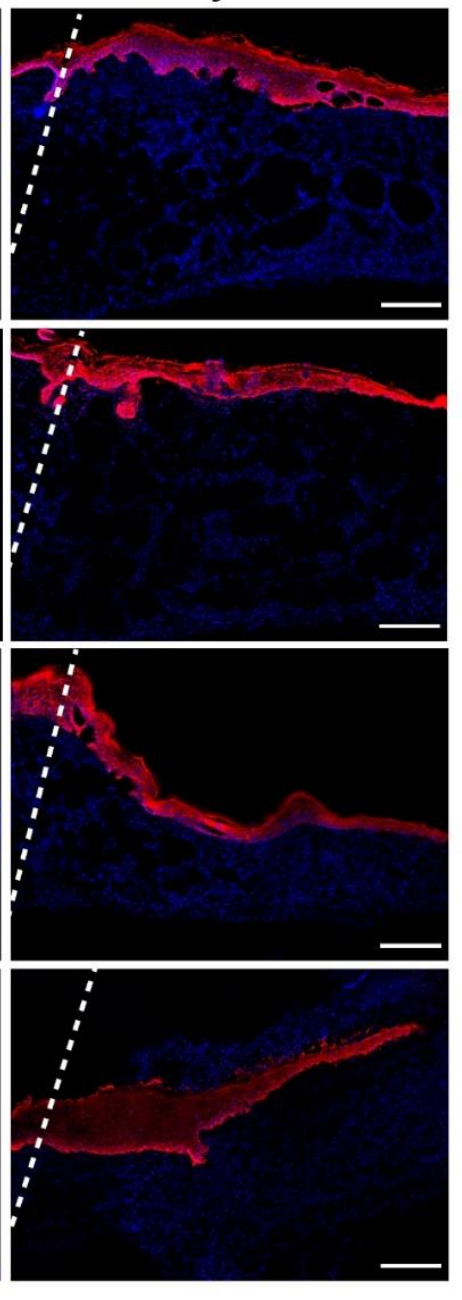

Figure 3. Epidermal Regeneration in a Diabetic Wound Healing Model. A) Four treatment conditions were evaluated in a mouse diabetic wound healing model at Day 3 and Day 7. B) Epidermal tongue length was quantified at Day 3 to determine extent of reepithelialization. C) Epidermal thickness was quantified for all at Day 7 to compare stages of healing. D) Representative images of the four treatment groups keratin-14 staining at Day 3 and Day 7 . Wound edges marked by dashed line. All graphs show mean $+/-$ standard deviation. Statistics: ANOVA, Multiple comparisons post-hoc tests (Tukey HSD). N=6. 


\section{Heparin $\mu$ lslands promote enhanced re-epithelialization in diabetic wounds}

Diabetic wounds are characterized by their inability to move from the inflammatory to proliferation phase of wound healing, marked by the onset of re-epithelialization and re-vascularization. We chose two time points for this diabetic wound healing study, Day 3 and Day 7, to further characterize the progression of wound healing. The four treatment groups for this study were MAP gel with $\mu$ lsands $\left(10 \% \mathrm{Hep}_{\text {High }}\right)$, MAP gel without ulslands as a material platform control, Oasis wound matrix as an advanced clinical control (currently approved for use in diabetic wounds), and Aquaphor as a basic clinical control (OTC wound hydration product) (Fig. 3A). Quantification of wound re-epithelialization (determined by regenerated epidermal tissue or "tongues") showed the $\mu$ lslands group improved wound closure relative to all other groups (Fig. 3B,D). This data aligned with gross observations of wound granulation taken at Days 3 and 7 (Supplemental Fig. 12). By Day 7 the majority of wounds for all groups except Aquaphor had fully re-epithelialized the wound bed (Supplemental Fig. 13), as determined by Keratin-14 staining, and therefore epidermal thickness was quantified as a measure of dermal regeneration. The $\mu$ lslands demonstrated a significantly greater epidermal thickness compared to the MAP and Oasis groups (Fig. 3C,D). In concert, these results demonstrate that the $\mu$ lslands treatment provided a more regenerative and accelerated early healing result compared to all controls.
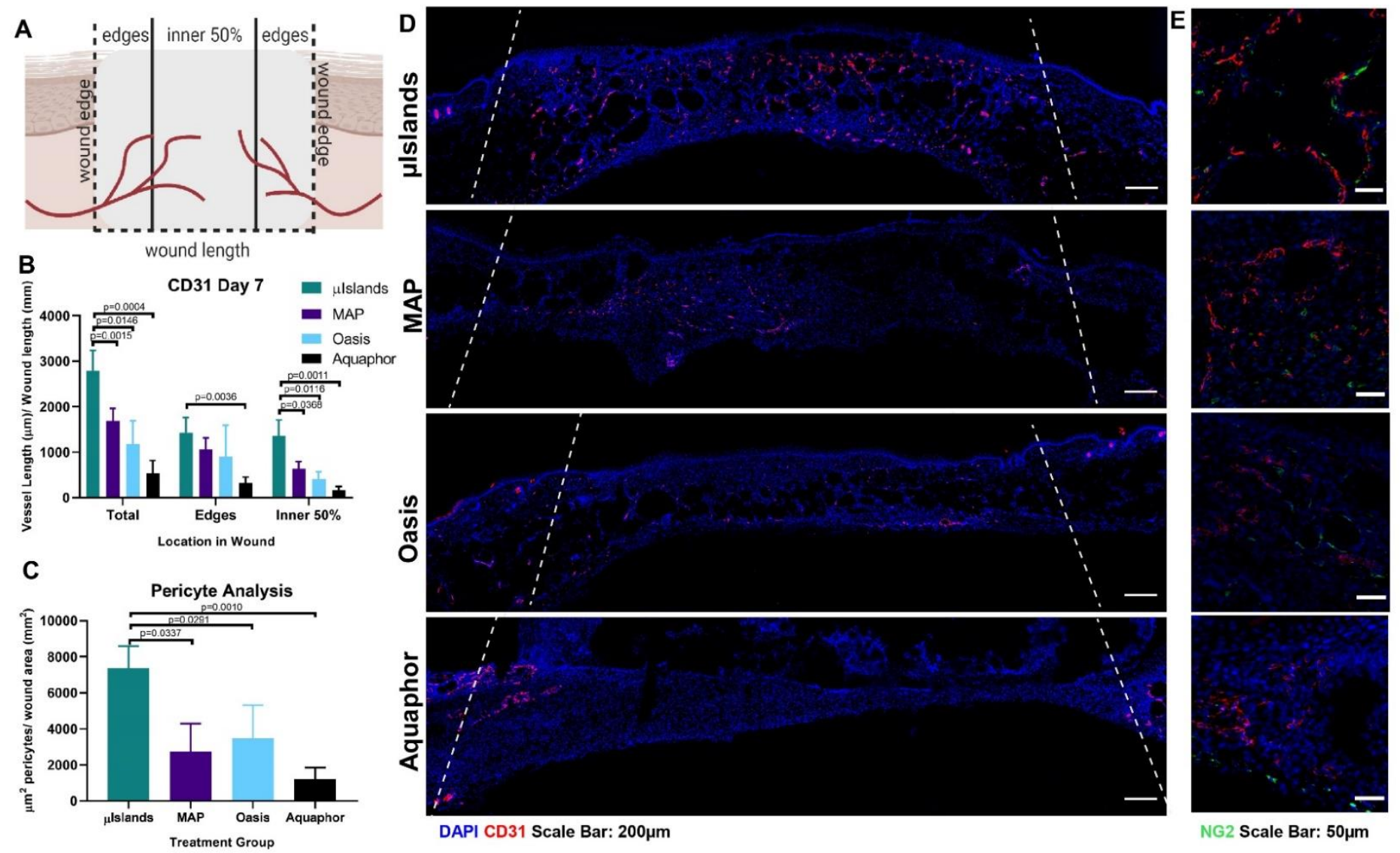

NG2 Scale Bar: $\mathbf{5 0 \mu m}$

Figure 4. Vascularization at Day 7 in a Diabetic Wound Healing Model. A) Vessel analysis was performed to determine the extent of vascularization throughout the wound. B) Vessel length was quantified for the middle and edges of the wound at Day 7 as determined by CD31 staining. C) Pericyte analysis was performed to assess the presence of mature vasculature as indicated by proximal NG2 staining to a blood vessel. D) Representative images of the four treatment groups CD31 staining Day 7 . Wound edges marked by dashed line. E) All graphs show mean +/- standard deviation. Statistics: ANOVA, Multiple comparisons post-hoc tests (Tukey HSD). $\mathrm{N}=6$.

\section{Uniform revascularization at Day 7}

Heparin's known interaction with key growth factors in angiogenesis and the importance of re-vascularization to wound healing prompted us to investigate vascularization among the groups at Day 7. At Day 7, the $\mu$ lslands group was characterized by the presence of extensive vasculature throughout the entire wound, while other groups had noticeably more of their re-vascularization occurring proximal to the wound edges (Fig. 4D). Quantification of this difference determined that $\mu$ lslands provided significantly more staining overall for CD31+ 
endothelial cells and that the distribution of blood vessels was equal between the inner $50 \%$ of the wound and the edges (Fig. 4B). Interestingly, when analysis is limited to the edges of the wound, there were no significant differences in re-vascularization among MAP, Oasis, and the $\mu$ lslands (Fig. 4B). By contrast, when analyzing the inner $50 \%$ of the wound, only the $\mu$ lslands show as much re-vascularization as was present in the outer wound area. The Aquaphor group had minimal vessels observed within the wound. As an additional metric to characterize the new vasculature, we stained for pericytes (an indicator for vessel maturity) by staining for $\mathrm{NG}^{+}$cells proximal ( $<5 \mu \mathrm{m}$ distant) to endothelial cells. Quantification of pericytes per wound area showed significantly more pericyte staining in the $\mu$ lslands group, indicating more mature vasculature (Fig. 4C,E). In summary, $\mu$ lslands produce a more mature and extensive vascular network throughout the entire diabetic wound by Day 7 .

A
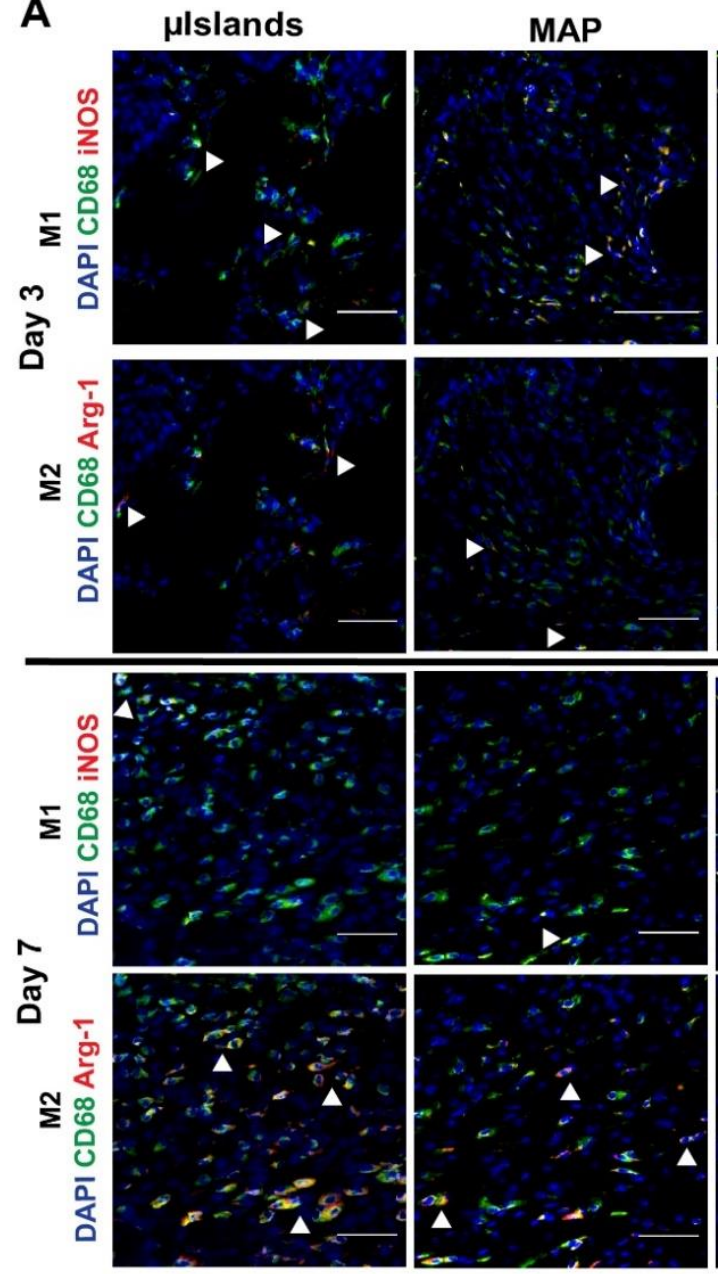
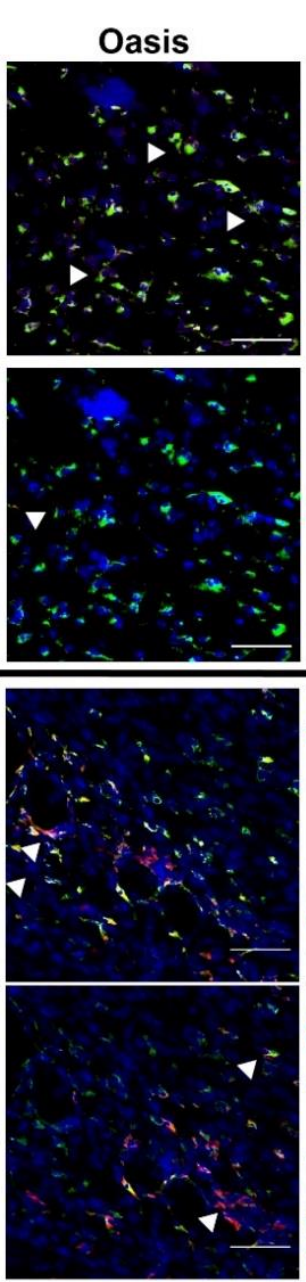
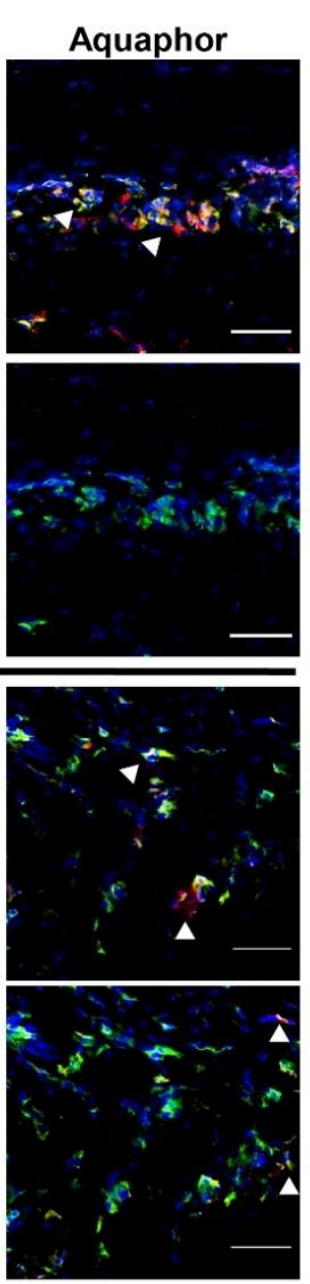
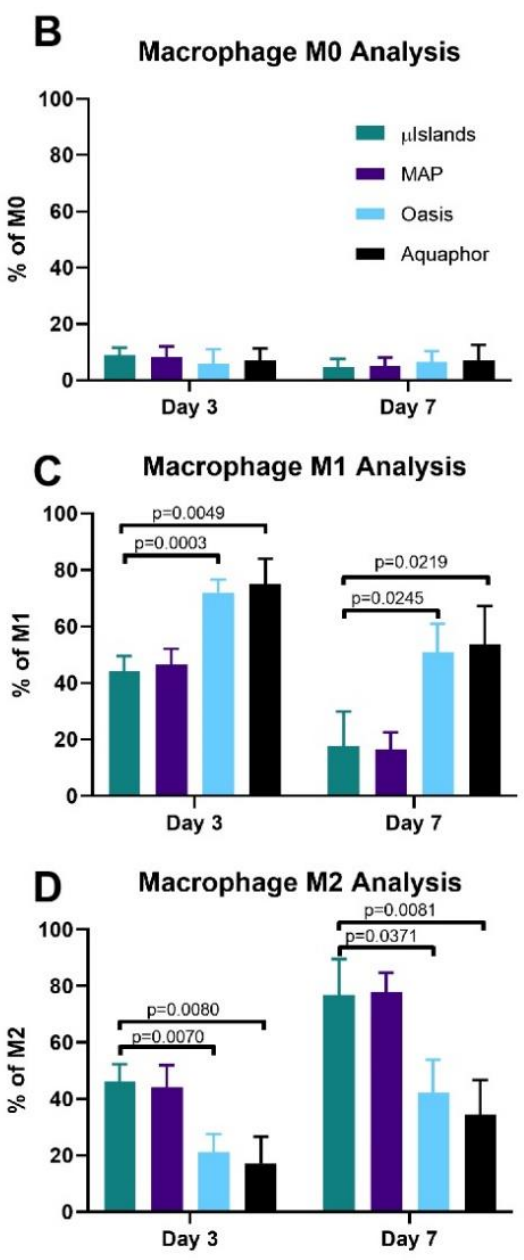

Figure 5. Macrophage Polarization. A) Representative images at Day 3 and Day 7 of macrophage M1 and M2 polarizations. B) Percentage of macrophages in the wounds for each group at Day 3 and 7 that only stained positive for CD68. C) Percentage of macrophages in the wounds that stained positive for the M1 inflammatory phenotype as indicated by CD68+ and iNOS+. D) Percentage of macrophages in the wounds that stained positive for the M2 pro-regenerative phenotype as indicated by CD68+ and Arg-1+. Statistics: ANOVA, Multiple comparisons post-hoc tests (Tukey HSD). N=6.

\section{No difference in immune modulation between $\mu$ Islands and MAP}

To investigate the potential for altered immune response caused by the presence of heparin $\mu$ lslands, we stained for macrophage polarization at Days 3 and 7 . There were no significant differences between any of the four groups for density of macrophages within the wounds (Supplemental Fig. 19). While we observed no difference between MAP with or without heparin $\mu$ lslands, we did observe that MAP groups (with and without heparin $\mu$ Islands) provided a clear immunomodulatory effect on wound macrophage polarization compared to either the Oasis or Aquaphor treatments (Fig. 5). Specifically, both MAP groups promoted more M2 than M1 polarization for both time points compared to Oasis and Aquaphor, and by Day 7 all macrophages were 
overwhelmingly M2 phenotype for both MAP conditions (Fig. 5A,C,D). Notably, these results aligned well with prior investigations of precast porous hydrogel scaffolds that are the geometric inverse of MAP gel ${ }^{24,25}$. Combined, these data demonstrate that the improved wound closure and re-vascularization effects observed for the heparin $\mu$ lslands group was likely not due to changes to the immune response within the wound.

\section{Conclusions}

Here we present a new type of bioactive scaffold which can harness local growth factors to form microscale chemotactic gradients, eliminating the need for exogenous delivery while maintaining an instructional microenvironment. Previously, creating gradients at this scale within biomaterials has required advanced biofabrication strategies (photolithography, bioprinting) that limits injectability as well as the ability to fill large wounds. MAP with heparin $\mu$ lslands significantly improved diabetic wound healing outcomes compared to an unaltered MAP gel and two clinically relevant control groups. We plan to use future studies to further understand the in vivo mechanism of improved healing by identifying and quantifying the cytokines being organized by the bioactive heparin $\mu$ slands. The presented approach has high translational potential for applications requiring quick tissue integration in challenging tissue environments.

\section{Acknowledgements}

Figure schematics created with BioRender.com. LP was supported by a National Science Foundation Graduate Research Fellowship and by the National Heart, Lung, and Blood Institute of the National Institutes of Health under Award Number F31HL154731. This work was partially supported through the US National Institutes of Health High Priority, Short-Term Project Award (1R56DK126020-01) and The Wallace H. Coulter Translational Partners Program at The University of Virginia.

\section{References}

1. Greenhalgh, D. G. The Role of Growth Factors in Wound Healing. Journal of Trauma and Acute Care Surgery 41, 159-167 (1996).

2. Briquez, P. S., Hubbell, J. A. \& Martino, M. M. Extracellular Matrix-Inspired Growth Factor Delivery Systems for Skin Wound Healing. Advances in Wound Care 4, 479-489 (2015).

3. Laiva, A. L., O'Brien, F. J. \& Keogh, M. B. Innovations in gene and growth factor delivery systems for diabetic wound healing. Journal of Tissue Engineering and Regenerative Medicine 12, e296-e312 (2018).

4. Rao, S. S., Venkatesan, J., Prabhu, A. \& Rekha, P. D. Natural polymeric biomaterials in growth factor delivery for treating diabetic foot ulcers. Journal of Drug Delivery Science and Technology 55, 101385 (2020).

5. Pop, M. A. \& Almquist, B. D. Biomaterials: A potential pathway to healing chronic wounds? Experimental Dermatology 26, 760-763 (2017).

6. Futrega, K., King, M., Lott, W. B. \& Doran, M. R. Treating the whole not the hole: necessary coupling of technologies for diabetic foot ulcer treatment. Trends in Molecular Medicine 20, 137-142 (2014).

7. Sakiyama-Elbert, S. E. Incorporation of heparin into biomaterials. Acta Biomaterialia 10, 1581-1587 (2014).

8. Liang, Y. \& Kiick, K. L. Heparin-functionalized polymeric biomaterials in tissue engineering and drug delivery applications. Acta Biomater. 10, 1588-1600 (2014).

9. Capila, I. \& Linhardt, R. J. Heparin-Protein Interactions. Angewandte Chemie International Edition 41, 390-412 (2002).

10. Singh, M., Berkland, C. \& Detamore, M. S. Strategies and Applications for Incorporating Physical and Chemical Signal Gradients in Tissue Engineering. Tissue Engineering Part B: Reviews 14, 341-366 (2008).

11. Nguyen, E. H., Schwartz, M. P. \& Murphy, W. L. Biomimetic Approaches to Control Soluble Concentration Gradients in Biomaterials. Macromolecular Bioscience 11, 483-492 (2011). 
12. Griffin, D. R., Weaver, W. M., Scumpia, P. O., Di Carlo, D. \& Segura, T. Accelerated wound healing by injectable microporous gel scaffolds assembled from annealed building blocks. Nature Materials 14, 737-744 (2015).

13. Turner, N. J. \& Badylak, S. F. The Use of Biologic Scaffolds in the Treatment of Chronic Nonhealing Wounds. Adv Wound Care (New Rochelle) 4, 490-500 (2015).

14. Kasiewicz, L. N. \& Whitehead, K. A. Recent advances in biomaterials for the treatment of diabetic foot ulcers. Biomater Sci 5, 1962-1975 (2017).

15. National Diabetes Statistics Report, 2017.

16. Singh, N., Armstrong, D. G. \& Lipsky, B. A. Preventing foot ulcers in patients with diabetes. JAMA 293, 217-228 (2005).

17. Parmaksiz, M., Dogan, A., Odabas, S., Elçin, A. E. \& Elçin, Y. M. Clinical applications of decellularized extracellular matrices for tissue engineering and regenerative medicine. Biomed. Mater. 11, 022003 (2016).

18. Michaels, J. et $\mathrm{al}$. $\mathrm{db} / \mathrm{db}$ mice exhibit severe wound-healing impairments compared with other murine diabetic strains in a silicone-splinted excisional wound model. Wound Repair Regen 15, 665-670 (2007).

19. Niezgoda, J. A., Van Gils, C. C., Frykberg, R. G., Hodde, J. P. \& Group, O. D. U. S. Randomized Clinical Trial Comparing OASIS Wound Matrix to Regranex Gel for Diabetic Ulcers. Advances in Skin \& Wound Care 18, 258-266 (2005).

20. Rutte, J. M. de, Koh, J. \& Carlo, D. D. Scalable High-Throughput Production of Modular Microgels for In Situ Assembly of Microporous Tissue Scaffolds. Advanced Functional Materials 0, 1900071.

21. Pfaff, B. N. et al. Selective and Improved Photoannealing of Microporous Annealed Particle (MAP) Scaffolds. bioRxiv 2020.10.27.358127 (2020) doi:10.1101/2020.10.27.358127.

22. Rydergren, S. Chemical Modifications of Hyaluronan using DMTMM-Activated Amidation. (Uppsala Universitet, 2013).

23. Nandi, S. \& Brown, A. C. Characterizing Cell Migration Within Three-dimensional In Vitro Wound Environments. JoVE (Journal of Visualized Experiments) e56099 (2017) doi:10.3791/56099.

24. Sussman, E. M., Halpin, M. C., Muster, J., Moon, R. T. \& Ratner, B. D. Porous Implants Modulate Healing and Induce Shifts in Local Macrophage Polarization in the Foreign Body Reaction. Ann Biomed Eng 42, 1508-1516 (2014).

25. Ratner, B. D. A pore way to heal and regenerate: 21 st century thinking on biocompatibility. Regen Biomater 3, 107-110 (2016).

26. Pomin, V. H. NMR Chemical Shifts in Structural Biology of Glycosaminoglycans. Anal. Chem. 86, 6594 (2014).

27. Zuo, H. et al. Regeneration of mature dermis by transplanted particulate acellular dermal matrix in a rat model of skin defect wound. J Mater Sci: Mater Med 23, 2933-2944 (2012).

28. Barbosa, I. et al. Improved and simple micro assay for sulfated glycosaminoglycans quantification in biological extracts and its use in skin and muscle tissue studies. Glycobiology 13, 647-653 (2003).

29. Hahn, M. S., Jao, C. Y., Faquin, W. \& Grande-Allen, K. J. Glycosaminoglycan composition of the vocal fold lamina propria in relation to function. Ann. Otol. Rhinol. Laryngol. 117, 371-381 (2008).

30. Pfisterer, L. \& Korff, T. Spheroid-Based In Vitro Angiogenesis Model. in Angiogenesis Protocols (eds. Martin, S. G. \& Hewett, P. W.) 167-177 (Springer, 2016). doi:10.1007/978-1-4939-3628-1_11.

31. Dunn, L. et al. Murine Model of Wound Healing. JoVE (Journal of Visualized Experiments) e50265 (2013) doi:10.3791/50265.

32. Corliss, B. A. et al. REAVER: A program for improved analysis of high-resolution vascular network images. Microcirculation 27, e12618 (2020). 